\title{
Combination of Lateral and PA View Radiographs to Study Development of Knee OA and Associated Pain
}

DOI:

$10.1117 / 12.2254295$

Document Version

Final published version

Link to publication record in Manchester Research Explorer

\section{Citation for published version (APA):}

Minciullo, L., Thomson, J., \& Cootes, T. (2017). Combination of Lateral and PA View Radiographs to Study Development of Knee OA and Associated Pain. In Medical Imaging 2017: Computer-Aided Diagnosis (Vol. 10134). (SPIE Proceedings). SPIE. https://doi.org/10.1117/12.2254295

\section{Published in:}

Medical Imaging 2017: Computer-Aided Diagnosis

\section{Citing this paper}

Please note that where the full-text provided on Manchester Research Explorer is the Author Accepted Manuscript or Proof version this may differ from the final Published version. If citing, it is advised that you check and use the publisher's definitive version.

\section{General rights}

Copyright and moral rights for the publications made accessible in the Research Explorer are retained by the authors and/or other copyright owners and it is a condition of accessing publications that users recognise and abide by the legal requirements associated with these rights.

\section{Takedown policy}

If you believe that this document breaches copyright please refer to the University of Manchester's Takedown Procedures [http://man.ac.uk/04Y6Bo] or contact uml.scholarlycommunications@manchester.ac.uk providing relevant details, so we can investigate your claim.

\section{OPEN ACCESS}




\title{
Combination of Lateral and PA View Radiographs to Study Development of Knee OA and Associated Pain
}

\author{
Luca Minciullo ${ }^{\mathrm{a}}$, Jessie Thomson ${ }^{\mathrm{a}}$, and Timothy F. Cootes ${ }^{\mathrm{a}}$ \\ ${ }^{a}$ Centre for Imaging Sciences, The University of Manchester, Stopford Building, Manchester, \\ UK
}

\begin{abstract}
Knee Osteoarthritis (OA) is the most common form of arthritis, affecting millions of people around the world. The effects of the disease have been studied using the shape and texture features of bones in PosteriorAnterior (PA) and Lateral radiographs separately. In this work we compare the utility of features from each view, and evaluate whether combining features from both is advantageous. We built a fully automated system to independently locate landmark points in both radiographic images using Random Forest Constrained Local Models. We extracted discriminative features from the two bony outlines using Appearance Models. The features were used to train Random Forest classifiers to solve three specific tasks: (i) OA classification, distinguishing patients with structural signs of OA from the others; (ii) predicting future onset of the disease and (iii) predicting which patients with no current pain will have a positive pain score later in a follow-up visit. Using a subset of the MOST dataset we show that the PA view has more discriminative features to classify and predict OA, while the lateral view contains features that achieve better performance in predicting pain, and that combining the features from both views gives a small improvement in accuracy of the classification compared to the individual views.
\end{abstract}

Keywords: Knee Osteoarthritis, Appearance Models, Combination of Views, Constrained Local Models

\section{INTRODUCTION}

Osteoarthritis $(\mathrm{OA})$ is the most common form of arthritis, affecting millions of people around the world, the chance of developing the desease being particularly high in older people. It has been reported ${ }^{1}$ that by 2030 around $20 \%$ of the American population will be above the age of 65 , and that half of them (35 million of patients) will be at high risk of developing OA, requiring a large amount of public money ${ }^{2}$ for treatments and surgery.

The most common signs of OA are: osteophytes, bony spurs that grow on the bones of the spine or around the joints, joint space narrowing (JSN) and calcium deposits. Painkillers and lifestyle changes are the only therapies currently available and eventually most patients have to undertake a total or partial joint arthoplasty.

$\mathrm{OA}$ is currently assessed from radiographs using the Kellgren and Lawrence (KL) ${ }^{3}$ grades from 0 to 4 , where 0 represents normality and 4 the most severe stage of OA. When a radiograph is taken clinicians assign a discrete KL grade based on features in the image. This is time consuming, subjective and there are shortages of suitably trained radiologists. There is an increasing need for reliable systems that can perform the grading automatically. Detecting knee OA and assessing its severity are crucial step for clinical decision making and a reliable prediction of the disease progression.

Current automated systems focus on the PA view, but research ${ }^{4}$ indicates the lateral knee view adds information about pain, prediction of disease and other measures. It also allows better analysis of disease by capturing features missed in the PA angle.

We have developed fully automated systems to analyse the shape and texture of bones in both lateral and PA knees. The goal of this work is to compare which view gives the most informative features for studying OA, and to explore whether better results can be achieved by combining information from both views.

Further author information: (Send correspondence to Luca Minciullo)

Luca Minciullo: E-mail: luca.minciullo@postgrad.manchester.ac.uk 
This work follows two different ones ${ }^{5,6}$ done in automated OA diagnosis. In the first one the authors built the first automated system to classify OA using lateral knee radiographs. This system was built on the MOST dataset, the only large dataset associated to a longitudinal study where at each visit both lateral and PA radiographs are acquired. This work showed that shape features extracted from lateral radiographs have promising discriminative capabilities, but lacked of a direct comparison with PA radiograph on the same dataset. The second work studied Posterior-Anterior (PA) knee radiographs from the OAI dataset ${ }^{7}$ to retrieve shape and texture features. They used a RFCLM on a 74 points model and extracted features of tibial texture.

Other approaches as the ones of ${ }^{8}$ Shamir and ${ }^{9}$ Anifah, where image processing techniques are applied to PA knee radiographs: in the former the authors extracted image content descriptors and image transforms to use as features in a Nearest Neighbor setting; the latter applied the unsupervised self organizing maps based on Gabor filter to classify the K-L grades. In the work of Jin ${ }^{10}$, the authors used medical infrared thermography of the $\mathrm{PA}$ view to extract features on which train a SVM classifier.

Our work aims at combining features from both lateral and PA knee radiographs. For both lateral and PA radiographs the method was the following: we manually annotated a few hundred images with a set of landmark points, obtaining a collection of discrete shapes, from which we built statistical shape and appearance models. We used a Random Forest Regression Voting Constrained Local Model (RFCLM) ${ }^{11-13}$ to locate points in both single bones and combinations of bones. The detection of a ROI containing the joint was done using an object detector based on Random Forests (RF) to automatically initialise the RFCLM on each image.

Once an automated annotation was found, we extracted shape, texture and appearance parameters and combined them to solve three tasks related with OA: (i) OA classification, distinguishing patients with structural signs of OA from the others; (ii) predicting future onset of the disease and (iii) predicting which patients with no current pain will have a positive pain score later in a follow-up visit.

\section{METHODS}

Our lateral knee model is made of four different sub-shapes: the patella (21 points), the lateral femoral condyle (24 points), the medial femoral condyle (25 points) and the tibia (32 points). We considered the lateral femur as the union of the two femoral condyles (49 points). The whole knee model is then made of 102 points (Figure 1). In this work we ignored the points of the patella because there was not a corresponding model for the PA view.

On the other hand, the PA model is made of two shapes: the femur and the tibia (37 points each, for a total of 74 points).
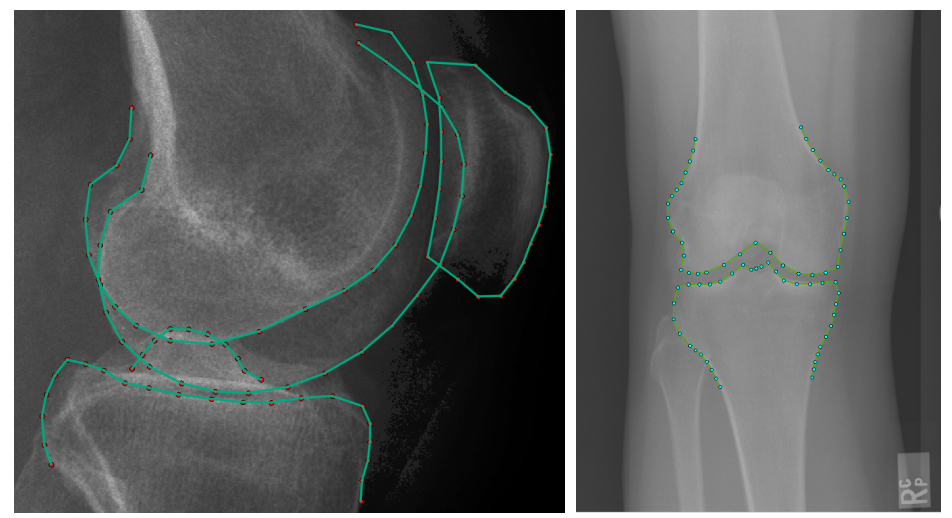

Figure 1. An example of the landmark points used to build the two shape models.

\subsection{Appearance Model}

The way we extracted features was by building an appearance model. Combined Appearance Models $(\mathrm{CAM})^{14}$ are an attempt to a better use of textural information and they are based on a statistical model that uses shape 
as one of its components. In this way we achieve better representation power compared to a shape model and this could bring more robustness. Such a model incorporates non redundant information of the shape and the texture of the object of interest. They are built by first retrieving a statistical shape model of the knee.

A shape model is a mathematical object that represents each shape $x=\left(x_{1}, y_{1}, x_{2}, y_{2}, \ldots\right)^{T}$ in the following way

$$
x=T\left(\bar{x}+P_{s} b_{s} ; t\right)
$$

where $\bar{x}$ is a representation of the mean shape in a suitable reference frame, $P_{s}$ is a matrix containing a set of modes of variation and $T$ applies a global similarity transformation with parameters $t$.

The shape parameters $b_{s}$ can be calculated from $x$ using

$$
b_{s}=P_{s}^{T}\left(T^{-1}(x ; t)-\bar{x}\right) .
$$
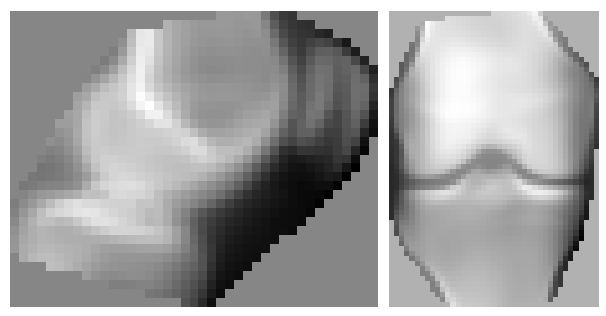

Figure 2. The mean appearance of the lateral (left) and Posterior-Anterior (right) models.

In order to build an appearance model we start by assuming that we have built a shape model of variation, as described in equation 1. Then we warp each of the training image to match the mean shape and we sample the texture information from the resulting objects. To minimise the effect of lighting variation, we normalise the samples using a scale factor $\alpha$ and an offset parameter $\beta$ so that the mean of the pixel values is zero and the sum of their squares is unity. After applying PCA to the set of vectors obtained we end up with the linear model that follows

$$
g=T\left(\bar{g}+P_{g} b_{g} ; t\right)
$$

where $\bar{g}$ is the mean grey-level vector, $P_{h}$ is a matrix of eigenvectors, explaining the textural variation and $b_{g}$ are texture parameters. The number of texture parameters was chosen to be constantly equal to 30 .

An Appearance model can be calculated by concatenating the two models and then via a further PCA. This is because shape and texture are often correlated.

$$
b=\left(\begin{array}{c}
W_{s} b_{s} \\
b_{g}
\end{array}\right), \quad b=Q c
$$

where $W_{s}$ is a diagonal matrix of weights, the shape eigenvalues, for each shape parameter, $b_{g}$ are the texture parameters and $c$ are the appearance parameters with corresponding eigenvectors listed in $Q$.

\subsection{Object Detection and Shape Model Matching}

The first step in the segmentation of the bones was to build a global searcher able to find the individual bones within each image. Our implementation used a Hough Forest approach. ${ }^{15}$ We defined a bounding box starting from a pair of landmark points and then sampled from each image a set of patches with different displacements, angles and scales with respect to the location of the bone of interest. We then trained a Random Forest to learn the functional relation between the pixel intensities in the image patches and the corresponding displacements. This RF is scanned over a new image at multiple scales and orientations, voting for likely knee locations. The output of the global search is a bounding box with two reference points, from which we initialise each model (Figure 3). 


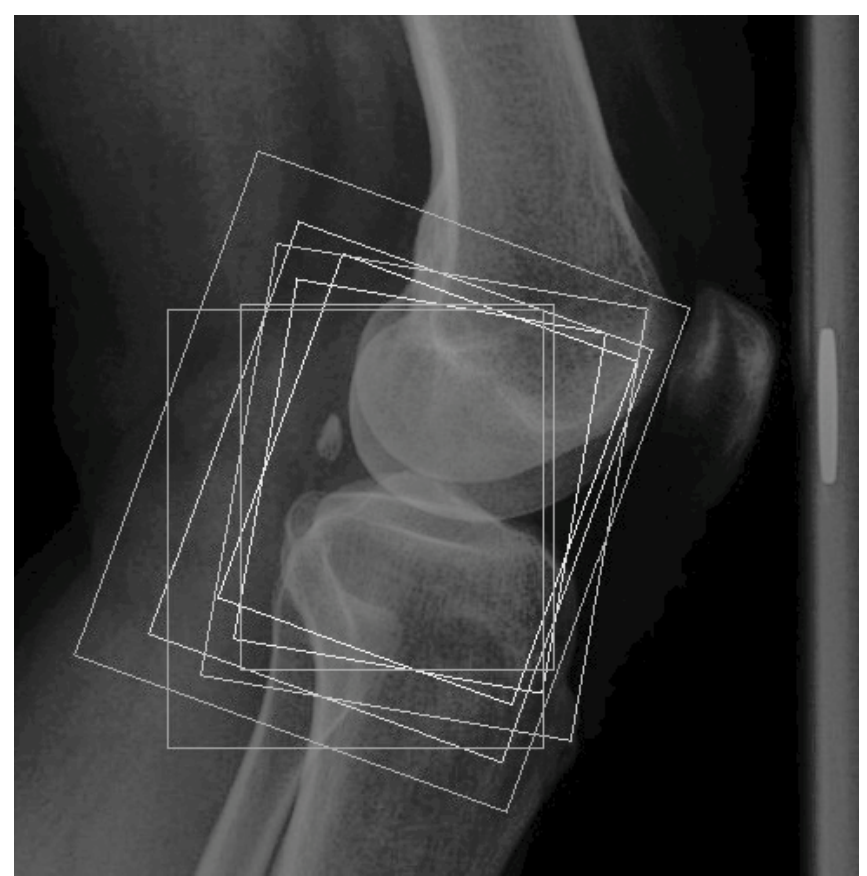

Figure 3. An example of the bounding boxes found by the Random Forest bone detector.

In the second step we improve the fitting of the model, by applying a sequence of increasingly refined Constrained Local Models. The idea is to independently train a point detector per landmark point. Each model uses regression-voting trees to predict point displacements from patches of image texture and constrains the points using a shape model. The algorithm has been used previously to find hips and knees ${ }^{16}$ from radiographs.

In the search phase we sample a set of patches around the current approximation of the point location. We feed those patches into the Random Forest, receiving a prediction per patch and tree for the location of the landmark point of interest. We combine all the predictions in a voting image $V_{i}()$ for each point $i$. The shape model is used to regularise the result, finding the parameters $b, t$ which maximise the total votes $Q(b, t)=\sum_{i=1}^{n} V_{i}\left(T\left(\bar{x}_{i}+P_{i} b_{i} ; t\right)\right)$. With regard to the lateral view, we used a different segmentation model for each of the three shapes involved. Each of those models involved a sequence of three increasingly refined CLM, with frame width equal to 50, 100 and 200 pixels. The frame widths associated to the PA radiographs were 50, 200 and 500, the first two aim to find the whole knee shape at once while the last one improving the fitting of tibia and femur separately.

The RFCLM is trained on manually annotated points placed around the shape outlines. We used 500 images from the MOST dataset to train the lateral RFCLM and 500 images from the OAI dataset for the PA model.

Table 1. Number of features per each shape and feature type.

\begin{tabular}{ccccccc}
\hline & Lateral view & \multicolumn{5}{c}{ PA view } \\
\hline & Shape & Texture & App. & Shape & Texture & App. \\
\hline Femur & 30 & 30 & 50 & 18 & 30 & 37 \\
\hline Tibia & 20 & 30 & 42 & 19 & 30 & 40 \\
\hline Knee & 44 & 30 & 55 & 35 & 30 & 51 \\
\hline
\end{tabular}

\subsection{Extraction and combination of the features}

The approach above enables us to fully automatically segment the outlines of the bones in new images. We can find shape, texture and appearance parameters given the points from the statistical shape model. 
In this work we explore how the parameters from different structures and views can be combined to most effectively classify the disease or pain status of the knee. To combine parameter vectors we simply concatenate them.

\section{RESULTS}

The results shown in this section are obtained performing a 5 -fold cross validation. For all the classification tasks introduced above we trained a Random Forest made of 25 trees. We show the performance of our technique by giving the areas under the Receiver Operating Characteristic (ROC) curves (AUC) for each bone and view or combinations of features.

\subsection{Data}

The images were taken from the Multicentre Osteoarthritis Study (MOST) dataset. This is a longitudinal prospective study that collected data from 3026 participants across with a 7-year follow-up. The data used in this work only considers data up to 30 months after baseline (second visit). Lateral and PA radiographs have been collected at each time-point for both knees. For the binary classification task the grades have been split into two groups: non-OA, KL $(0,1)$, and the OA group, KL(2-4). KL grades and reported pain within the last 30 days are used as outcomes in the experiments, and different subsets of the data are used to solve the tasks of interest: (i) OA classification, using $4628 \mathrm{OA}(K L \geq 2)$ and 6805 non-OA images; (ii) OA prediction, using 3234 baseline images with no OA $(K L \leq 1)$ of those 272 develop OA within 30 months and 2962 do not develop OA; (iii) pain prediction, 845 knees with no pain at baseline with 478 later developing pain and 367 not developing pain.

\subsection{OA Classification}

The results of the automated diagnosis are shown in Table 2. In general, features extracted from the PA view perform better than those of the Lateral view. Furthermore, in various instances by combining features of the two views achieved AUCs higher than both the individual models. However, the best overall AUC of the combined model is only as good as the best AUC of the PA model, in both cases corresponding to the appearance features of the full knee model.

Table 2. Binary OA classification. AUC for the two individual views and their concatenation.

\begin{tabular}{|c|c|c|c|c|c|c|c|c|c|}
\hline \multicolumn{3}{|c|}{ Lateral view } & \multicolumn{3}{|c|}{ PA view } & \multicolumn{4}{|c|}{ Lateral + PA views } \\
\hline & Shape & Texture & App. & Shape & Texture & App. & Shape & Texture & App. \\
\hline Femur & $74 \pm 0.1$ & $80.9 \pm 0.01$ & $82.3 \pm 0.01$ & $78.7 \pm 0.1$ & $87.1 \pm 0.1$ & $87.3 \pm 0.1$ & $81 \pm 0.1$ & $87.9 \pm 0.1$ & $87.7 \pm 0.1$ \\
\hline Tibia & $72.3 \pm 0.01$ & $79.2 \pm 0.01$ & $81 \pm 0.2$ & $78.4 \pm 0.2$ & $89 \pm 0.1$ & $88.9 \pm 0.1$ & $80.5 \pm 0.1$ & $89.1 \pm 0.1$ & $89 \pm 0.01$ \\
\hline Knee & $81.3 \pm 0.2$ & $82.7 \pm 0.01$ & $85.3 \pm 0.2$ & $89.6 \pm 0.1$ & $89.4 \pm 0.1$ & $90.4 \pm 0.1$ & $89.7 \pm 0.2$ & $89.6 \pm 0.1$ & $90.5 \pm 0.01$ \\
\hline
\end{tabular}

\subsection{OA Prediction}

The accuracy of predicting future onset of structural OA are, shown in the following table. Again, in most cases

Table 3. Prediction of future onset of OA. AUC for the two individual views and their concatenation.

\begin{tabular}{|c|c|c|c|c|c|c|c|c|c|}
\hline \multicolumn{3}{|c|}{ Lateral view } & \multicolumn{3}{|c|}{$\mathrm{PA}$ view } & \multicolumn{4}{|c|}{ Lateral + PA views } \\
\hline & Shape & Texture & App. & Shape & Texture & App. & Shape & Texture & App. \\
\hline Femur & $57 \pm 0.6$ & $57.4 \pm 0.8$ & $58 \pm 0.8$ & $57.5 \pm 0.1$ & $62.4 \pm 1.2$ & $60.3 \pm 0.8$ & $58.9 \pm 0.5$ & $63.1 \pm 1.1$ & $60.2 \pm 1.2$ \\
\hline Tibia & $54.1 \pm 1.3$ & $55.2 \pm 1$ & $53.1 \pm 0.1$ & $59.6 \pm 0.2$ & $65.1 \pm 0.4$ & $64.3 \pm 1.2$ & $59.7 \pm 0.8$ & $62.6 \pm 1$ & $60.4 \pm 0.5$ \\
\hline Knee & $54.8 \pm 0.8$ & $56.3 \pm 0.5$ & $56.6 \pm 1.1$ & $60.3 \pm 0.5$ & $64 \pm 0.6$ & $63 \pm 0.5$ & $60.2 \pm 0.8$ & $63.2 \pm 0.9$ & $62.1 \pm 0.6$ \\
\hline
\end{tabular}


the PA view has better performance than the lateral view, though the difference is much smaller than in the previous experiments. Moreover, features from the combined model are often more discriminative than the ones of the individual models achieving the best overall result, though by a small margin.

\subsection{Pain Prediction}

Predicting future onset of knee joint pain is the most challenging of the three tasks. Pain scores are very subjective and finding patterns in the way pain develops with time has proven to be extremely difficult. Results on this task are shown in Table 4

Table 4. Prediction of future pain. AUC for the two individual views and their concatenation.

\begin{tabular}{cccccccccc}
\hline \multicolumn{3}{l}{ Lateral view } & & PA view & & & \multicolumn{2}{c|}{ Lateral + PA views } \\
\hline & Shape & Texture & App. & Shape & Texture & App. & Shape & Texture & App. \\
\hline Femur & $53.6 \pm 1.1$ & $54.9 \pm 1.7$ & $\mathbf{5 5 . 7} \pm \mathbf{1 . 3}$ & $48.3 \pm 0.8$ & $\mathbf{5 6 . 8} \pm \mathbf{1 . 3}$ & $53.8 \pm 0.1$ & $52.6 \pm 1.1$ & $\mathbf{5 6 . 9} \pm \mathbf{0 . 1}$ & $55.1 \pm 0.2$ \\
\hline Tibia & $54.1 \pm 0.5$ & $53.5 \pm 1.2$ & $55.4 \pm 0.2$ & $51.5 \pm 1.2$ & $52.9 \pm 1.3$ & $53.3 \pm 1.2$ & $55.4 \pm 0.9$ & $55.6 \pm 1.6$ & $54.8 \pm 0.7$ \\
\hline Knee & $53.1 \pm 2.6$ & $54.7 \pm 0.8$ & $55.3 \pm 1.8$ & $48.5 \pm 1.1$ & $52.2 \pm 0.8$ & $52.4 \pm 1.7$ & $55.2 \pm 0.2$ & $54 \pm 0.3$ & $54.3 \pm 1.3$ \\
\hline
\end{tabular}

Unlike the previous experiments, features extracted from lateral knee radiographs show consistently better performance than the ones of the PA view. Furthermore, on average combining the two sets of features does not seem to increase the performance. Nevertheless, the best overall AUC $56.9 \pm 0.1$ is achieved by the combination of textures features of the femur.

\section{CONCLUSIONS AND FUTURE WORK}

In this work we have shown the first attempt at combining shape, texture and appearance parameters of radiographs of the knee joint acquired from different views. Our experiments show that such concatenation leads to improved accuracy in various tasks, though often by a small margin. As far as we are aware this work is the first large scale direct comparison of the two views when studying OA and its future development.

The results show that the combination of the two view contains more discriminative features, but the magnitude of the improvement in performance is not large. Future work will involve the development of alternative ways of combining features from the two views. For example, we will be investigating way of building a combined model for the appearance and apply redundancy reduction techniques. We also aim to design a deep learning architecture to merge the information coming from the radiographs.

It would also be interesting to use combined features to solve other OA related classification problems in the computer aided study of the disease such as: automated assessment of the severity of OA, detection of osteophytes severity and joint space narrowing.

\section{ACKNOWLEDGMENTS}

The research leading to this results has received funding from EPSRC Centre for Doctoral Training grant 1512584.

\section{REFERENCES}

[1] Felson, D. T., Niu, J., Neogi, T., Goggins, J., Nevitt, M., Roemer, F., Torner, J., Lewis, C., Guermazi, A., and Group, M. I., "Synovitis and the risk of knee osteoarthritis: the most study," Osteoarthritis and Cartilage 24(3), 458-464 (2016).

[2] Chen, A., Gupte, C., Akhtar, K., Smith, P., and Cobb, J., "The global economic cost of osteoarthritis: how the uk compares," Arthritis 2012 (2012).

[3] Kellgren, J. and Lawrence, J., "Radiological assessment of osteo-arthrosis," Annals of the rheumatic diseases 16(4), 494 (1957). 
[4] LaValley, M. P., McLaughlin, S., Goggins, J., Gale, D., Nevitt, M. C., and Felson, D. T., "The lateral view radiograph for assessment of the tibiofemoral joint space in knee osteoarthritis: its reliability, sensitivity to change, and longitudinal validity," Arthritis 83 Rheumatism 52(11), 3542-3547 (2005).

[5] Minciullo, L. and Cootes, T., "Fully automated shape analysis for detection of osteoarthritis from lateral knee radiographs," in [International Conference on Pattern Recognition], (To appear), IEEE (2016).

[6] Thomson, J., ONeill, T., Felson, D., and Cootes, T., "Automated shape and texture analysis for detection of osteoarthritis from radiographs of the knee," in [International Conference on Medical Image Computing and Computer-Assisted Intervention], 127-134, Springer (2015).

[7] Lester, G., "Clinical research in oa-the nih osteoarthritis initiative," J Musculoskelet Neuronal Interact 8(4), 313-314 (2008).

[8] Shamir, L., Ling, S. M., Scott Jr, W. W., Bos, A., Orlov, N., Macura, T. J., Eckley, D. M., Ferrucci, L., and Goldberg, I. G., "Knee x-ray image analysis method for automated detection of osteoarthritis," IEEE Transactions on Biomedical Engineering 56(2), 407-415 (2009).

[9] Anifah, L., Purnama, I. K. E., Hariadi, M., and Purnomo, M. H., "Osteoarthritis classification using self organizing map based on gabor kernel and contrast-limited adaptive histogram equalization," The open biomedical engineering journal 7, 18 (2013).

[10] Jin, C., Yang, Y., Xue, Z.-J., Liu, K.-M., and Liu, J., "Automated analysis method for screening knee osteoarthritis using medical infrared thermography," Journal of Medical and Biological Engineering 33(5), 471-477 (2013).

[11] Cootes, T. F., Ionita, M. C., Lindner, C., and Sauer, P., "Robust and accurate shape model fitting using random forest regression voting," in [European Conference on Computer Vision], 278-291, Springer (2012).

[12] Cristinacce, D. and Cootes, T. F., "Feature detection and tracking with constrained local models.," in $[B M V C], \mathbf{1}(2), 3$ (2006).

[13] Lindner, C., Thiagarajah, S., Wilkinson, J. M., Wallis, G. A., Cootes, T. F., arcOGEN Consortium, et al., "Fully automatic segmentation of the proximal femur using random forest regression voting," IEEE transactions on medical imaging 32(8), 1462-1472 (2013).

[14] Cootes, T. F., Taylor, C. J., et al., "Statistical models of appearance for computer vision," (2004).

[15] Gall, J. and Lempitsky, V., "Class-specific hough forests for object detection," in [Decision forests for computer vision and medical image analysis], 143-157, Springer (2013).

[16] Lindner, C., Thiagarajah, S., Wilkinson, J. M., Wallis, G. A., Cootes, T. F., arcOGEN Consortium, et al., "Accurate bone segmentation in $2 \mathrm{~d}$ radiographs using fully automatic shape model matching based on regression-voting," in [International Conference on Medical Image Computing and Computer-Assisted Intervention], 181-189, Springer (2013). 